\title{
Mathematical model of eyring fluid in a scraped surface heat exchanger
}

\author{
A Imran', AM Siddiqui' ${ }^{2}$, M Numan ${ }^{1}$, MA Rana ${ }^{3}$ and A Waheed ${ }^{1}$ \\ ${ }^{1}$ Department of Mathematics, COMSATS University Islamabad, Attock Campus, Kamra Road, Pakistan. \\ ${ }^{2}$ Department of Mathematics, York Campus, Pennsylvania State University, York, PA 17403, USA. \\ ${ }^{3}$ Department of Mathematics and Statistics, Riphah International University, Sector I-14, Islamabad, Pakistan.
}

Submitted: 22 February 2019; Revised: 26 February 2020; Accepted: 07 April 2020

\begin{abstract}
In this work, a novel mathematical model for steady incompressible isothermal flow of Eyring fluid in a scraped surface heat exchanger (SSHE) was explored. Foodstuff possesses non-Newtonian features, therefore for studying these effects, Eyring fluid model has been considered. For the analysis of flow inside SSHE, lubrication approximation theory (LAT) was exploited to model the flow, and exact solution is achieved. The flow about a sequence of pivoted scraper blade in a channel, in which one wall is moving and the other is at rest was analysed, and the pressure gradient applied transversely. Expressions for important flow indicators such as velocity profile, stream functions, pressures, flow rates and the probable regions of backward flow down the blades, the drag and lift forces on the blades and walls were computed. The impact of various flow parameters was exhibited graphically. It was observed that the velocity profile in all regions of SSHE increases with enhancement in the non-Newtonian parameter $\gamma$, whereas an opposite behaviour was recorded with rising value of $\beta$.
\end{abstract}

Keywords: Exact solution, eyring fluid, lubrication approximation theory.

\section{INTRODUCTION}

Scraped surface heat exchangers (SSHEs) play a vibrant role in the industries manufacturing various types of food stuff. They contribute to chemical industries for producing paints and removing wax from oil. These are also used in pharmaceutical industries. SSHEs are mainly used in the food industry to mix, cool or heat foodstuff and some other purification, gelatinisation and crystallisation processes. The composition of SSHE is shown in Figure 1.

Simple plate exchangers are used to process less viscous fluids, whereas SSHEs are employed to process highly viscous foodstuff. Foodstuff such as ice cream, chocolates, spreads, sauces, cream, purees, caramel and yoghurt are prepared using SSHEs. A SSHE primarily comprise a cylindrical rotating shaft that is termed as rotor and a hollow stationary cylinder that is termed as stator, that develop an annular section through which fluid is moved. Outer surface of the device works as the heat transfer surface and it is usually lying under another cylindrical tube, that creates a gap through which heating or cooling service fluid passes. The blades are installed in the rotor. These blades mix the foodstuff and remove the foodstuff that sticks at the inner boundary. A normal SSHE consists of four blades, which are designed at 90 degree apart, or two blades which are 180 degree apart, which are located periodically at the rotor. In order to improve power consumption, the blades are designed with holes in it and to remove channelling, oval shape stators are also used. The blades of SSHE perform several tasks which are very important in food processing. As mentioned earlier the main task is to improve the heat transfer between stators and to forward the fluid near the stator surface. This indicates that fluid to be more homogeneously processed and it avoids temperature in homogeneities. As the stator is

\footnotetext{
* Corresponding author (ali.imran@cuiatk.edu.pk; iD https://orcid.org/0000-0002-1820-6539)
} 
continuously scraped, which enhances the efficiency in heat transfer, it enables SSHE to work for longer span of time. In the mixing process, these blades help in producing a homogeneous quality and develop good taste and texture of the product. In the production of ice cream, these blades mix the ice and air particles, which are made on the cooled stator. The process of mixing the fluid during heating or cooling makes enough use of the high temperature gradient. To optimise the production run and decrease the cost of the product, large theoretical and experimental investigations have been carried out on various types of SSHEs using several fluid rheological models and working conditions. Due to the complex scenario of cooling/heating and mixing mechanism, there are various indicators that require attention in order to have a complete insight of the flow inside SSHE. First, the shape and working conditions of SSHE has a certain vital role, and other parameters, namely, device dimension, blade design, rotation speed of blade and flow rates require careful attention to enhance the efficiency and form suitable conditions inside the heat exchanger. Study of literature on SSHE reveals that extensive theoretical and experimental research has been carried out. Wang et al. (1999) reported the flow in a SSHE isothermally, and experimentally affirmed the results with MIR technique. Russell et al. (1997) investigated the flow of non-Newtonian fluid inside SSHE without considering heat involvement. Trommlene et al. (1971) and Qin et al. (2003) investigated heat transfer phenomena inside SSHE. In another study Trommlene et al. (1971) investigated the power consumption. Complete mathematical analysis of flow along with heat involvement in SSHE can be explored with the aid of numerical techniques. Sun et al. (2004) explored the flow with heat transfer effects by employing the numerical simulations. In another study, Sun et al. (2006) analysed the flow of fluid with decreasing viscosities in lid-driven cavities. Fitt and Please (2001) analysed the isothermal flow of shear thinning fluid in a simple model SSHE and found the requisite power usage in the process of rotation and pumping. Duffy et al. (2007) reported the flow of Newtonian fluid analytically under the isothermal conditions in slender gap SSHE. With the aid of lubrication approximation theory (LAT), they simplified the flow and obtained an exact solution for velocities, volume flow rates and forces on the wall and blades. They also found the probable position of the blades and determined that the blade will have ideal position with the inner wall when their pivots are placed near to the edge of the blades. Fitt et al. (2007) determined the process of channelling in a slender gap SSHE for a Newtonian fluid. Rodrigues et al. (2009) explored the work in a laboratory based SSHE and obtained qualitative match with numerical techniques extracted through lattice Boltzman discretisation techniques. Smith et al. (2010) studied the heat transfer mechanism by using Newtonian fluid in SSHE. They explored the flow with temperature dependent viscosity. Siddiqui et al. (2015) studied the magnetic properties of Newtonian fluid in SSHE. Ishigaki (2018) carried out an investigation for the motion of a compressible Oldroyd fluid model in an infinite layer. For the case of suitable external force, the problem possesses solution of parallel flow type. Endo et al. (2017) demonstrated the flow of viscoelastic fluid for the case of two-dimensional layer domain and obtained $\mathrm{L}^{2}$-stability for the Poiseuille-type flow. Imran et al. $(2016 ; 2017)$ studied the flow of non-Newtonian fluid in SSHE.

\section{METHODOLOGY}

Let us take a steady case of incompressible Eyring fluid flow in a channel with width $H$, where there is an arrangement of inclinely balanced thin blades. The surface along $y=0$ is in motion with velocity $U$, and the other surface along $y=H$ is stationary. Assuming that thin blades take position $0 \leq x \leq L$ installed at $\left(x_{p}, h_{p}\right)$, where $0 \leq x_{p} \leq L$ and $0 \leq h_{p} \leq H<<L$, and distance around blades $l$, the area $L \leq x \leq L+l$ represents full breadth of channel. This setting is repeated sequentially, with sequence $L+l$. The device width $\mathrm{H}$, the period, and speed may be interpreted in term of cylindrical coordinates as, $H=R_{2}-R_{1}, L+l=\frac{2 \pi R_{1}}{N} \quad$ and $U=R_{1} w$, here $R_{1}$ is the radius of rotor and $R_{2}$ is the radius of stator, $N$ denotes the count of blades, and $\omega$ represents angular speed of the rotor.

Let $\theta$ indicate inclination of the blade with the lower boundary and $y=h(x)$ represents the inclination height, which can be expressed as;

$h(x)=h_{p}+\theta\left(x-x_{p}\right)$.

Assuming $h_{0}=h(0)$ and $h_{1}=h(L)$ in a manner

$$
h_{0}=h_{p}-\theta x_{p}, h_{1}=h_{p}+\theta\left(L-x_{p}\right), \text { and } \theta=\frac{h_{1}-h_{0}}{L} .
$$

For steady flow, the blades of the device maintain equilibrium position along the forces because of the fluid, the pivot, and the boundary of the device. We will analyse the situation when the blades do not touch the wall of the device, in a manner $h_{0}>0, h_{1}<H$. 
Expressions for velocity, pressure, volume fluxes and stream function can be defined for different sections of SSHE as $u_{k} \mathbf{i}+v_{k} \mathbf{j}, p_{k}, Q_{k}$ and $\psi_{k}$, where $k=1,2,3$ indicate the three different stations as: section 1, $0 \leq x \leq L, 0 \leq y \leq h$ that is the region beneath the blade, $k=2$; section $2,0 \leq x \leq L, h \leq y \leq H$ this is the region on the upper side of blade and $k=3$; section $3, L \leq x \leq L+l, 0 \leq y \leq H$.

The equations of motion for the problem are;

$$
\boldsymbol{\nabla} \cdot \mathbf{V}=\mathbf{0} .
$$

$$
\rho \frac{d \mathbf{v}}{d t}=\operatorname{div} \mathbf{S}+\rho \mathbf{b},
$$

where,

$$
\mathbf{S}=\frac{\beta \operatorname{ArchSinh}\left(-\frac{1}{\gamma}\left|\mathbf{A}_{1}\right|\right)}{\left|\mathbf{A}_{1}\right|} \mathbf{A}_{1}
$$

where $\beta$ and $\gamma$ are representing the material constants, $\mathbf{A}_{1}$ the first Rivlin-Ericksen tensor is;

$$
\mathbf{A}_{1}=\nabla \mathbf{V}+(\nabla \mathbf{V})^{T}
$$

$\boldsymbol{\nabla V}$ is the velocity gradient. This model shows pseudoplastic behaviour at finite values of stress components. Further, this model changes in Newtonian fluid model when $\mu=\frac{\beta}{\gamma}$.

LAT describes the flow of fluid in a geometry in which one dimension is very small in comparison to others. In case of SSHE, different gaps are considered very small i.e., the aspect ratio being of order $10^{-1}$, the appropriately reduced Reynolds number being of order $10^{-2}$, so that LAT can be used to study the flow [Duffy et al. (2007), Smith et al. (2010)]

The governing equations using LAT i.e.,

$$
\frac{\partial}{\partial x}<<\frac{\partial}{\partial y} \text { and } v<<u
$$

give

$$
\begin{aligned}
& \mathbf{S}_{x y}=\mathbf{S}_{y x}=\beta \operatorname{ArchSinh}\left(-\frac{1}{\gamma} \frac{d u_{k}}{d y}\right) \\
& \mathbf{S}_{y z}=\mathbf{S}_{z y}=0 \\
& \frac{d}{d y}\left(\operatorname{ArchSinh}\left(-\frac{1}{\gamma} \frac{d u_{k}}{d y}\right)\right)=\frac{1}{\beta} \frac{\partial p_{k}}{\partial x}
\end{aligned}
$$

$\frac{\partial p_{k}}{\partial y}=0$

Equation (9) signifies that $p_{k} \neq p_{k}(y)$ means $p_{k}=p_{k}(x)$ only.

$\frac{d}{d y}\left(\operatorname{ArchSinh}\left(-\frac{1}{\gamma} \frac{d u_{k}}{d y}\right)\right)=\frac{1}{\beta} \frac{d p_{k}}{d x}$

and respective B.Cs for $k=1,2,3$ are,

$u_{1}=U$ at $y=0, u_{1}=0$, at $y=h$,

$u_{2}=0$ at $y=h, u_{2}=0$ at $y=H$,

in $0 \leq x \leq L$, and

$u_{3}=U$ at $y=0, u_{3}=0$ on $y=H$,

in $L \leq x \leq L+l$.

The non-dimensional parameters are defined as under;

$$
\begin{aligned}
& x=L \bar{x}, \quad y=h_{p} \bar{y} \quad \theta=\frac{h_{p} \bar{\theta}}{L}, \quad l=L \bar{l}, \\
& x_{p}=L \bar{x}_{p}, \quad H=h_{p} \bar{H}, h_{0}=h_{p} \bar{h}_{0}, \quad h_{1}=h_{p} \bar{h}_{1}, u_{k}=U \bar{u}_{k}, \\
& p_{k}=\frac{\mu U L}{h_{p}^{2}} \bar{p}_{k}, F_{y}=\frac{\mu U L}{h_{p}^{2}} \bar{F}_{y}, \quad \beta=\frac{\mu U \bar{\beta}}{h_{p}}, \gamma=\frac{U \bar{\gamma}}{h_{p}}
\end{aligned}
$$

Omitting the bar sign on dimensionless quantities,

$\frac{d}{d y}\left(\operatorname{ArchSinh}\left(-\frac{1}{\gamma} \frac{d u_{k}}{d y}\right)\right)=\frac{1}{\beta} \frac{d p_{k}}{d x}$,

the respective no slip B.Cs are,

$u_{1}=1$ at $y=0, u_{1}=0$, at $y=h$

$u_{2}=0$ at $y=h, u_{2}=0$ at $y=H$,

in in $0 \leq x \leq 1$, and

$u_{3}=1$ at $y=0, u_{3}=0$ at $y=H$.

in $1 \leq x \leq 1+l$.

The solution is obtained by integrating equation (15) twice for $k=1,2,3$ w.r.t. equations (16) - (18), whereas 
the values of constant integration $\gamma_{1}, \gamma_{2}, \delta_{1}, \delta_{2}, \phi_{1}$, and $\phi_{2}$ are determined by using Mathematica. While finding these constants of integration in each region after using boundary conditions we have obtained four sets of roots in which three are extraneous. For the solution in each region we have included one set of constant of integration values, which satisfies the boundary conditions.

$$
\begin{aligned}
& u_{1}=\gamma_{2}-\frac{\beta \gamma \operatorname{Cosh}\left[\frac{p_{1 x} y}{\beta}+\gamma_{1}\right]}{p_{1 x}}, \\
& u_{2}=\delta_{2}-\frac{\beta \gamma \operatorname{Cosh}\left[\frac{p_{2 x} y}{\beta}+\delta_{1}\right]}{p_{2 x}}, \\
& u_{3}=\phi_{2}-\frac{\beta \gamma \operatorname{Cosh}\left[\frac{p_{3 x} y}{\beta}+\phi_{1}\right]}{p_{3 x}} .
\end{aligned}
$$

Now to determine the volume flow rate in different parts of SSHE, consider,

$Q_{1}=\int_{0}^{h} u_{1} d y$,

or

$$
\begin{aligned}
& Q_{1}=h \gamma_{2}+\frac{\beta^{2} \gamma\left(\operatorname{Sinh}\left[\gamma_{1}\right]-\operatorname{Sinh}\left[\frac{h p_{1 x}}{\beta}+\gamma_{1}\right]\right)}{p_{1 x^{2}}}, \\
& Q_{2}=\int_{h}^{H} u_{2} d y
\end{aligned}
$$

or

$Q_{2}=-h \delta_{2}+H \delta_{2}+\frac{\beta^{2} \gamma\left(\operatorname{Sinh}\left[\frac{h p_{2 x}}{\beta}+\delta_{1}\right]-\operatorname{Sinh}\left[\frac{H p_{2 x}}{\beta}+\delta_{1}\right]\right)}{p_{2 x}{ }^{2}}$
$Q_{3}=\int_{0}^{H} u_{3} d y$

$Q_{3}=H \phi_{2}+\frac{\beta^{2} \gamma\left(\operatorname{Sinh}\left[\phi_{1}\right]-\operatorname{Sinh}\left[\frac{H p_{3 x}}{\beta}+\phi_{1}\right]\right)}{p_{3 x^{2}}}$,

Exact expressions for pressure gradient are impossible to determine directly from equations (23-27). Using the value of $\gamma_{1}, \gamma_{2}, \delta_{1}, \delta_{2}, \phi_{1}$, and $\phi_{2}$ in equations (23-27) and expanding to first power of $p_{1 x}, p_{2 x}$, and $p_{3 x}$, respectively by capitalising the strength of Maclaurin series in Mathematica, after some simplification we have determined the following expressions for pressure gradient;

$p_{1 x}=\frac{12\left(3 h-2 Q_{1}\right) \beta}{h^{2}}$,

$p_{2 x}=\frac{24 Q_{2} \beta}{H^{3} \gamma}-\frac{216\left(Q_{2} \beta\right) h}{H^{4} \gamma}+\frac{2160 Q_{2} \beta h^{2}}{H^{5} \gamma}$,

$p_{3 x}=\frac{12\left(3 H-2 Q_{3}\right) \beta}{H^{2}}$.

We see that $p_{3 x}$ is independent of $x$; on the other hand $p_{1 x}$ and $p_{2 x}$ varies with $x$. In order to determine expressions for stream function we have;

$$
\frac{\partial \psi_{1}}{\partial y}=u_{1}, \frac{\partial \psi_{2}}{\partial y}=u_{2}, \psi_{1}=0 \text { at } y=0, \psi_{2}=Q_{1} \text { at } y=h,
$$

in $0 \leq x \leq 1$, and

$\frac{\partial \psi_{3}}{\partial y}=u_{3}, \psi_{3}=0$ at $y=0$,

in $1 \leq x \leq 1+l$, therefore,

$$
\begin{gathered}
\psi_{1}=\frac{p_{1 x}^{2} y \gamma_{2}-\beta^{2} \gamma \operatorname{Cosh}\left[\gamma_{1}\right] \operatorname{Sinh}\left[\frac{p_{1 x} y}{\beta}\right]+\beta^{2} \gamma \operatorname{Sinh}\left[\gamma_{1}\right]-\beta^{2} \gamma \operatorname{Cosh}\left[\frac{p_{1 x} y}{\beta}\right] \operatorname{Sinh}\left[\gamma_{1}\right]}{p_{1 x^{2}}} \\
\psi_{2}=\frac{p_{2 x}{ }^{2}\left(Q_{1}+(-h+y) \delta_{2}\right)+\beta^{2} \gamma\left(\operatorname{Sinh}\left[\frac{h p_{2 x}}{\beta}+\delta_{1}\right]-\operatorname{Sinh}\left[\frac{p_{2 x} y}{\beta}+\delta_{1}\right]\right)}{p_{2 x^{2}}{ }^{2}}
\end{gathered}
$$


$\psi_{3}=\frac{p_{3 x}^{2} y \phi_{2}-\beta^{2} \gamma \operatorname{Cosh}\left[\phi_{1}\right] \operatorname{Sinh}\left[\frac{p_{3 x} y}{\beta}\right]+\beta^{2} \gamma \operatorname{Sinh}\left[\phi_{1}\right]-\beta^{2} \gamma \operatorname{Cosh}\left[\frac{p_{3 x} y}{\beta}\right] \operatorname{Sinh}\left[\phi_{1}\right]}{p_{3 x^{2}}{ }^{2}}$

From conservation of mass

$$
Q_{1}+Q_{2}=Q_{3}
$$

in line with the evidence that the wall $y=H$ comprise of the streamline $\psi_{2}=Q_{1}+Q_{2}$ in $0 \leqslant x<1$ and $\psi_{3}=Q_{3}$ in $1 \leqslant x \leqslant 1+l$.

Equation (9) shows that the pressure is not the function of $y$. Assuming pressure to be uniform at the edge of the blades so that,

$$
p_{1}(1)=p_{2}(1)=p_{3}(1)=p_{L}
$$

and

$$
p_{1}(0)=p_{2}(0)=p_{3}(1+l)=p_{0}
$$

$$
p_{1}=\frac{-24 h Q_{1} \beta+24 h_{1} Q_{1} \beta-36 h h_{1} \beta \log \left(\frac{h}{h_{1}}\right)}{h h_{1} \theta}+p_{L}
$$

Putting $x=0$ in equations (37-38) and $x=1+l$ in equation (39), and using equation (36), we get three expressions of $p_{0}-p_{L}$,

$$
\begin{aligned}
p_{0}-p_{L} & =\frac{-24 h_{0} Q_{1} \beta+24 h_{1} Q_{1} \beta+36 h_{0} h_{1} \beta \log \left[h_{0}\right]-36 h_{0} h_{1} \beta \log \left[h_{1}\right]}{h_{0} h_{1} \theta}, \quad \ldots(40) \\
p_{0}-p_{L} & =\frac{24 H^{2} h_{0} Q_{2} \beta-108 H h_{0}{ }^{2} Q_{2} \beta+720 h_{0}{ }^{3} Q_{2} \beta-24 H^{2} h_{1} Q_{2} \beta+108 H h_{1}{ }^{2} Q_{2} \beta-720 h_{1}{ }^{3} Q_{2} \beta}{H^{5} \theta \gamma},
\end{aligned}
$$

$$
p_{0}-p_{L}=\frac{12\left(3 H-2 Q_{3}\right) \beta}{H^{2}} l
$$

\section{Qualitative features of the flow}

From equations (19-21) we conclude that the flow behaviour in region 2 is same whereas there might be back flow in region 1 under the blades and in region 3. Due to adverse pressure gradient, back flow may take place in region 1 . Most probably velocity component $u_{1}$ may be equal to zero on the blade but also near the curve $y=y_{01}(x)$. Now in order to determine the region $u_{1}=0$ along the blade $y=h$ representing $y=y_{01}(x)$

$$
y_{01}=\frac{-\beta \gamma_{1}-\beta \operatorname{ArcCosh}\left[\frac{p_{1 x} \gamma_{2}}{\beta \gamma}\right]}{p_{1 x}}
$$

In region between the blades i.e., region 3 flow is rectilinear, and streamlines are parallel to the channel walls (this is quite evident from plots of $\psi_{3}$ in Figures 5 and 6 . It is quite evident from equation (42) that 
$p_{0}>p_{L}$ in such a way that $Q_{3}<\frac{1}{2} H$ and $p_{3 x}<0$ which means that pressure gradient is adverse and back flow may occur. Further, equation (21) indicates at the point $y=y_{03} \neq H$ where $u_{3}=0$ and has expression,

$y_{03}=\frac{-\beta \phi_{1}-\beta \operatorname{ArcCosh}\left[\frac{p_{3 x} \phi_{2}}{\beta \gamma}\right]}{p_{3 x}}$,

and $y=y_{m 3}$ when $u_{3 y}=0$ is found as

$y_{m 3}=-\gamma \operatorname{Sinh}\left[\frac{p_{3 x} H}{\beta}+\phi_{1}\right]$,

therefore in $0<y_{03}<H$ and $0<y_{m 3}<H$.

\section{Drag and lift forces}

Drag $F_{x}$ and the lift $F_{y}$ forces acting in axial and transverse direction on the blades because of the fluid are determined as;

$$
\begin{aligned}
& F_{x}=-\int_{0}^{1}\left(\frac{\partial u_{1}}{\partial y}-\frac{\partial u_{2}}{\partial y}\right)_{y=h} d x-\theta \int_{0}^{1}\left(p_{1}-p_{2}\right) d x \\
& F_{y}=\int_{0}^{1}\left(p_{1}-p_{2}\right) d x \\
& F_{y}=\frac{12 \beta}{H^{5} h_{1} \theta \gamma}\left[( h _ { 0 } - h _ { 1 } ) ( h _ { 0 } - h _ { 1 } ) h _ { 1 } \left(H^{2}-3 H\left(h_{0}+2 h_{1}\right)\right.\right. \\
& \left.+15\left(h_{0}^{2}+2 h_{0} h_{1}+3 h_{1}^{2}\right)\right) Q_{2}+H^{5}\left(3 h_{1}+2 Q_{1}\right) \gamma+ \\
& \left.H^{5} h_{1}\left(3 h_{0}+2 Q_{1}\right) \gamma \log \left[\frac{h_{1}}{h_{0}}\right]\right]
\end{aligned}
$$

The force acting along the $x$-direction in region $0 \leq x \leq 1$ at $y=0$ because of the fluid is evaluated as;

$$
\begin{aligned}
& F_{0}=\int_{0}^{1}\left(\frac{\partial u_{1}}{\partial y}\right)_{y=0} d x+\int_{1}^{1+l}\left(\frac{\partial u_{3}}{\partial y}\right)_{y=0} d x \\
& F_{0}=\frac{1}{h_{0}{ }^{2} h_{1}{ }^{2} \gamma^{2}} 12\left(1 9 2 ( h _ { 0 } - h _ { 1 } ) \left(27 h_{0}{ }^{2} h_{1}{ }^{2}+36 h_{0} h_{1} Q_{1}{ }^{2}\right.\right. \\
&\left.-4\left(h_{0}+h_{1}\right) Q_{1}{ }^{3}\right)+h_{0}{ }^{2} h_{1}{ }^{2}\left(6 h_{0}-h_{0}{ }^{3}+h_{0}{ }^{2} Q_{1}\right. \\
&\left.+h_{1}\left(-6+h_{1}{ }^{2}-h_{1} Q_{1}\right)\right) \gamma^{2}+h_{0}{ }^{2} h_{1}{ }^{2}\left(h_{0}{ }^{3}-\right. \\
&\left.h_{0}{ }^{2} Q_{1}+h_{1}{ }^{2}\left(-h_{1}+Q_{1}\right)\right) \gamma^{3}-4 h_{0}{ }^{2} h_{1}{ }^{2} Q_{1}
\end{aligned}
$$




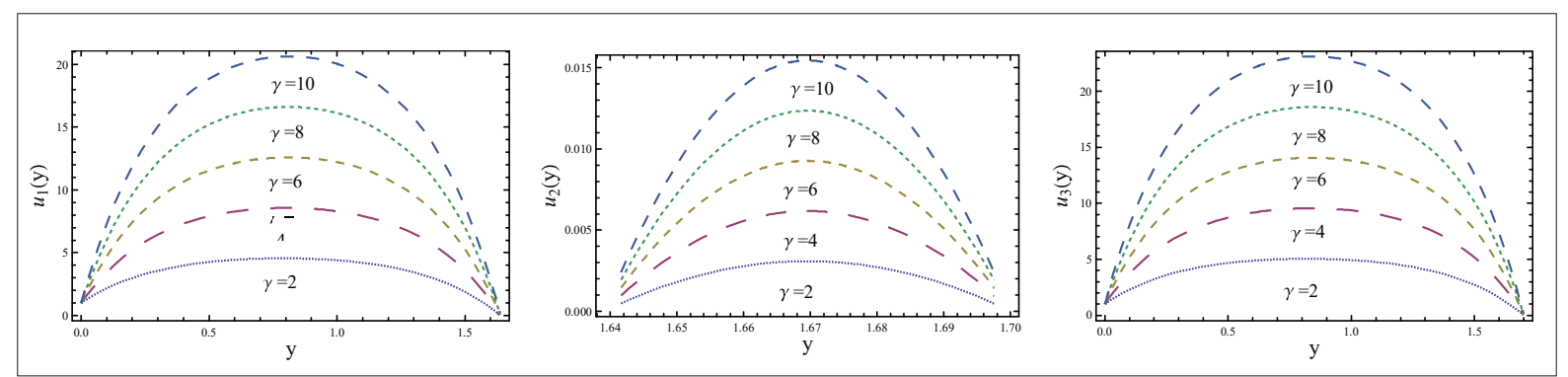

Figure 2: Impact of $\gamma$ with $H=1.7, l=2, x_{p}=0.49, \theta=1.25322, \beta=0.3, x=1, p_{1 x}=p_{2 x}=p_{3 x}=-1$
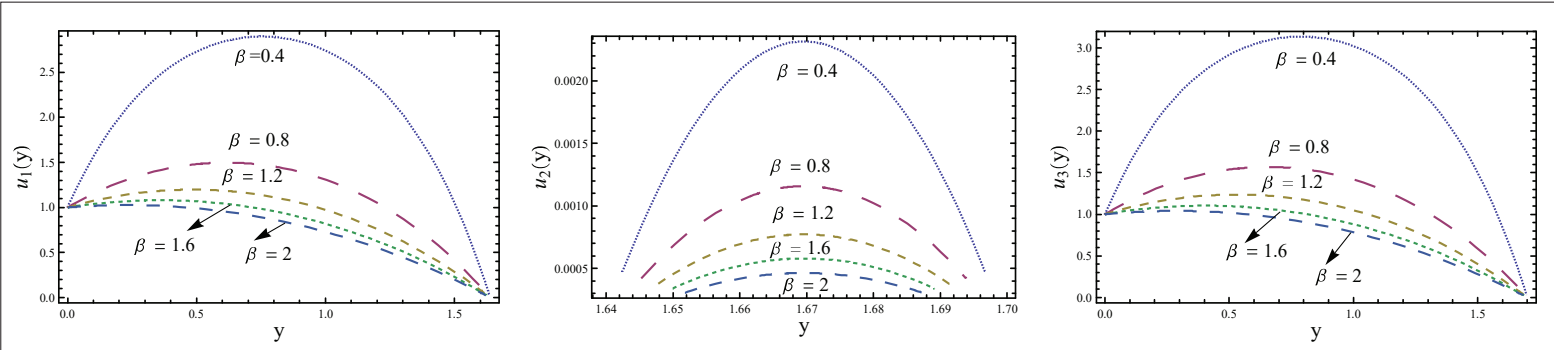

Figure 3: Impact of $\beta$ on velocity profile with $H=1.7, l=2, x_{p}=0.49, \theta=1.25322, x=1, p_{1 x}=p_{2 x}=p_{3 x}=-1$
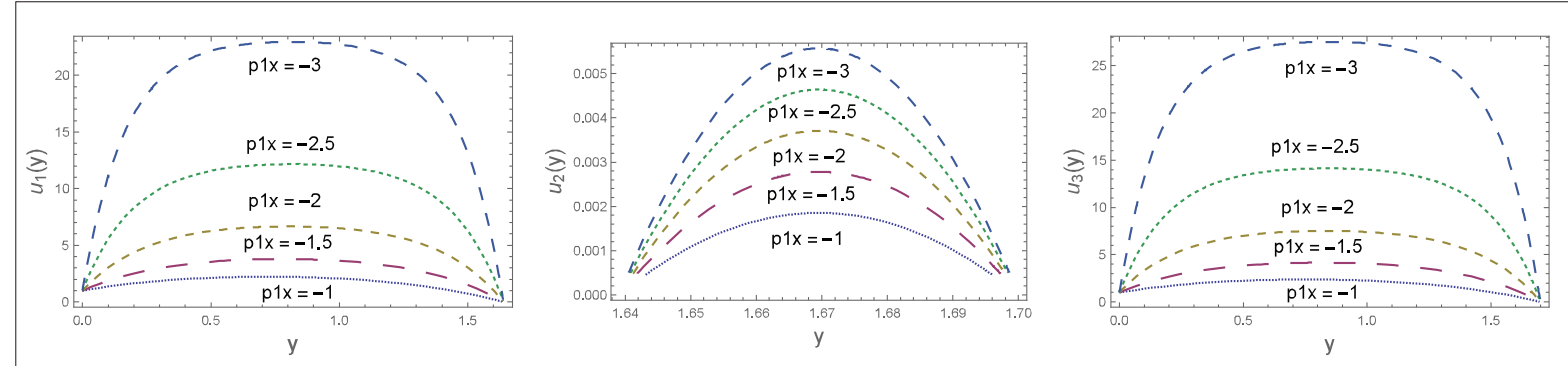

Figure 4: The effect of increasing pressure gradient on velocity profile with $H=1.7, \quad x_{p}=0.49, l=2, \theta=1.25322$, $\beta=0.5, \quad \gamma=2, x=1$

on the blades of the channel. An attempt was made to discuss the qualitative features of the flow, especially area of the back flow underneath the blades. Now, we will graphically study the variation of different flow indicators on the velocities, volume fluxes and on the stream functions. Figure 2 depicts the impact of $\gamma$ on the velocities. It is observed that velocity in region 1 , region 2 and region 3 increases with the rise in the value of non-Newtonian parameter $\gamma$. It is quite evident from
Figure 2 that the non-Newtonian parameters can be used to accelerate the transport phenomena. It is also observed that at $y=0$ the velocity is zero. As we move upward in the channel the velocity rises and has maximum value at the center of the channel. As we further move near the boundary $y=h$ the velocity becomes zero. Plots of increasing values of $\beta$ on the velocity profile are demonstrated in Figure 3. It is seen that as the value of $\beta$ is increased, the velocity profile in the stations of SSHE 


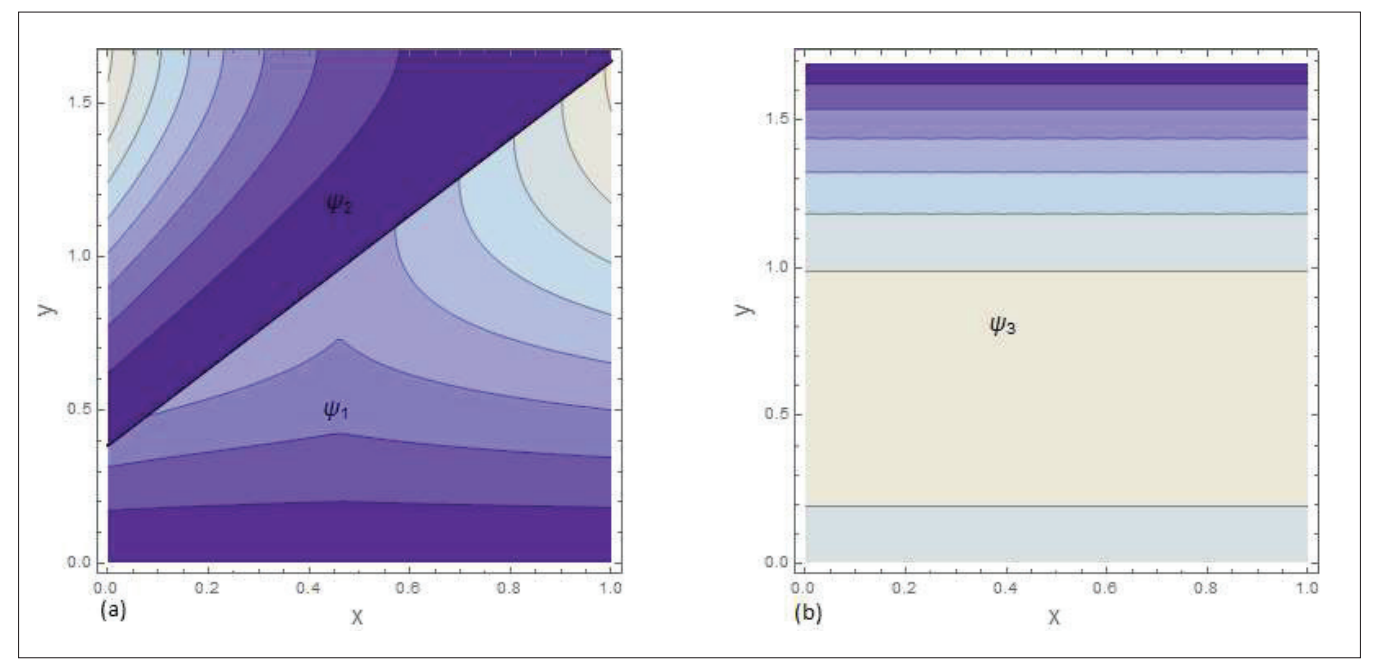

Figure 5: Graphs of stream lines: (a) below the thick line velocity distribution is illustrated in region 1 and above the thick line velocity distribution in region 2 ; (b) velocity distribution in region 3 with $H=1.7, l=2, x_{p}=0.49, \theta=1.25322, \gamma=2, \beta=0.5, x=1, p_{1 x}=p_{2 x}=p_{3 x}=-0.5$

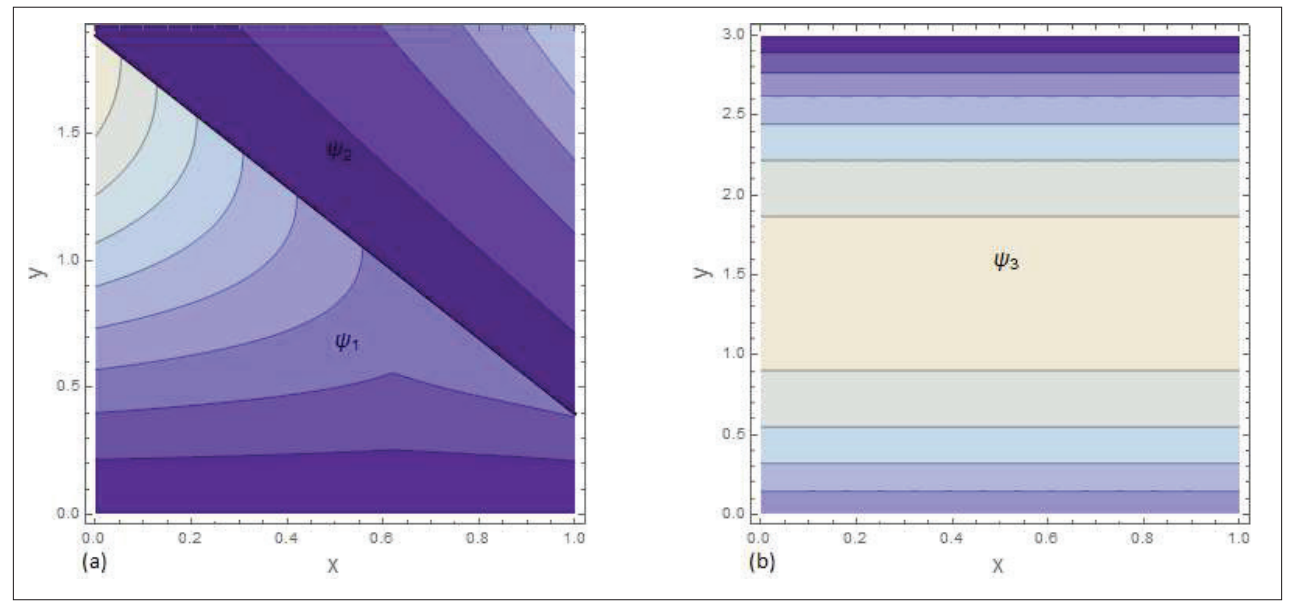

Figure 6: (a) Velocity distribution in region 1 and region 2; (b) streamline patterns in region 3 with $H=1.7, l=2, x_{p}=0.595, \theta=-1.48967, x=2, \quad p_{1 x}=p_{2 x}=p_{3 x}=0.5$

declines which means that the Eyring fluid parameter $\beta$ creates obstacle in the fluid motion. The plots of increasing pressure gradient on the velocity expressions are exhibited in Figure 4. It is worth to mention here that as the value of favourable pressure gradient is enhanced, velocity profile in all sections of SSHE is accelerated and this is the natural behaviour of fluid motion. It is a point to be noted here that in velocity plots, parabolic velocity profiles are obtained in various regions inside the SSHE, which are in line with the experimental studies obtained through MRI technique for SSHE geometry. Wang et al. (1999) investigated the two-dimensional flow in SSHE under isothermal scenario. They developed a theoretical model and experimentally armed their investigation using MRI technique. In the experimental studies, they observed parabolic velocity profile in SSHE. We have developed a mathematical model and it is quite evident from Figures 2-4 that we have obtained parabolic velocity profile through SSHE, which validates our mathematical study. Figures 5-6 demonstrate the velocity distribution inside SSHE. In Figures 5(a) and 6(a) the velocity distribution in region 1 and region 2 are exhibited. $\psi_{1}$ and $\psi_{2}$ reflect the streamline patterns in region 1 and 2 , respectively. The thick line divides both regions and 


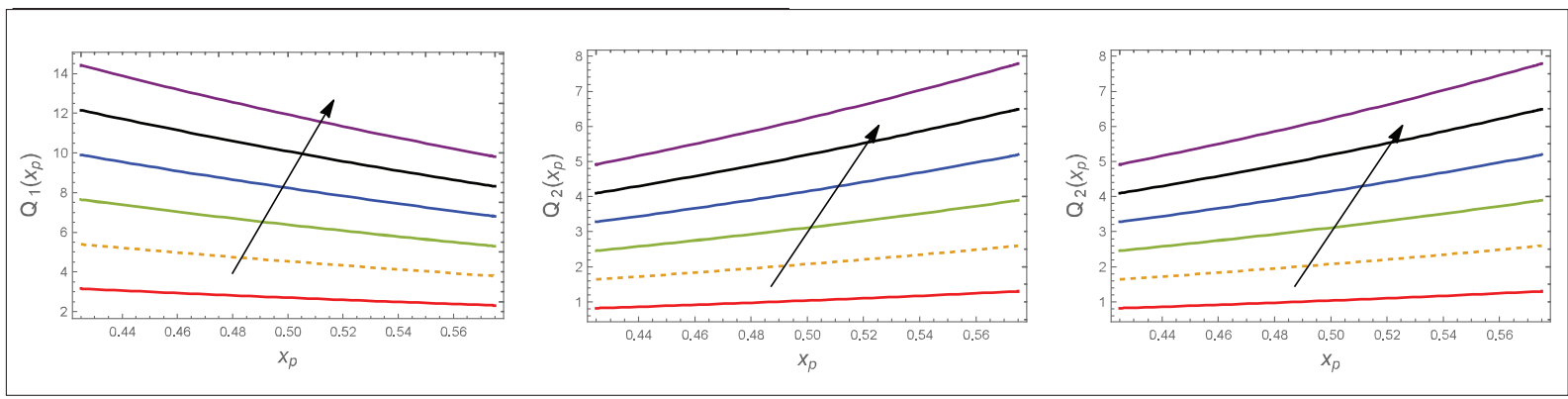

Figure 7: Flow rate variation with increasing values of $\gamma$ with $l=2, \theta=1.25322, H=3, \gamma=2,4,6,8,10,12, \quad p_{1 x}=$ $p_{2 x}=p_{3 x}=-1$

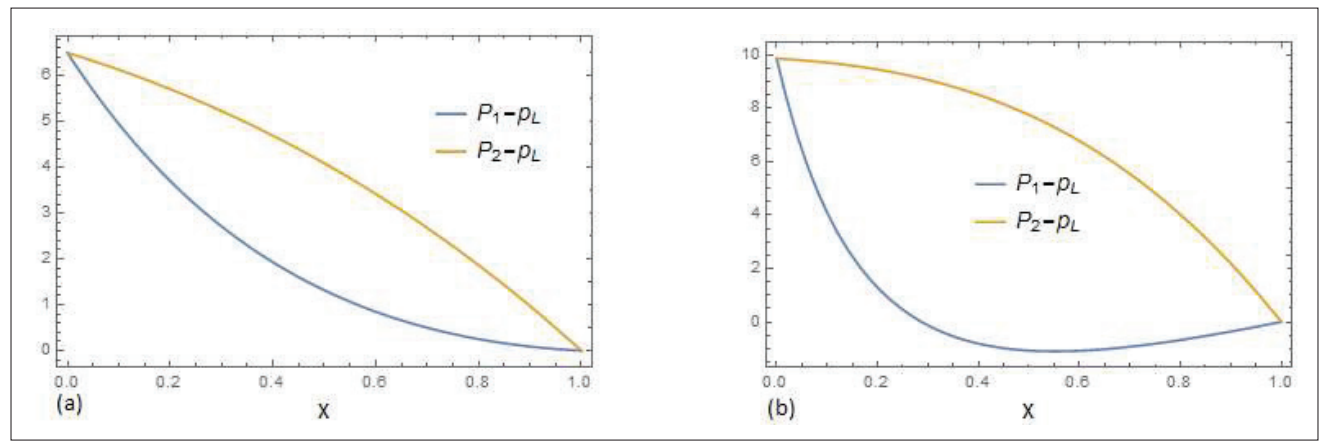

Figure 8: Plots of pressure at the edge of blades inside SSHE are demonstrated with (a) $H=1.7$, $l=2, \theta=0.5, \gamma=2, \beta=0.5$; (b) $H=1.7, l=2, \theta=1.25322, \gamma=2, \beta=0.5$

it is observed that there is a back flow under the blades. Figures 5(b) and 6(b) show the velocity distribution in region 3 for different values of angle of inclination of blade $\theta$. It is evident from these two figures that flow behaviour is rectilinear in this part of SSHE. Plots of flow rate are exhibited in Figure 7 for different values of $\gamma$. It is observed that flow rate is quite significantly enhanced by increasing values of Eyring fluid parameter $\gamma$. Figure 8 illustrates the plots of pressure rise $p_{1}-p_{L}$ and $p_{2}-p_{L}$ at the edge of the blades for different values of angle of inclination of blade. It is observed that, as the angle of inclination is enhanced, the gap between the curves of pressure at the edge of blade is inflated.

\section{CONCLUSION}

We investigated flow of Eyring fluid flow in SSHE. LAT was employed to simplify the equation of motion as these approximations are valid for studying flow inside SSHE. Solution for time independent flow of a Eyring fluid was obtained through a sequence of pivoted scraper blades.
The flow is due to motion of one wall and pressure gradient. The two-dimensional flow is investigated in a transverse portion of SSHE. Exact solution was obtained and expression for velocity profiles for various stations inside SSHE such as the probable regions of backward flow down the blades, flow rates, stream functions, drag and lift forces on the wall and on the blades are recovered. For the purpose of investigating impact of the different flow parameters, graphs for velocity profile, stream functions flow rate and pressure rise at the edge of the blades are incorporated. The same behaviour was attained through graphs as studied by Duffy et al. (2007), which confirms our investigation. This work provides quantitative insight of few fundamental characteristics of the non-Newtonian fluid flow through SSHE. It will be a basis for future investigations to include more complicated physical aspects. For onward investigations on SSHE it will be beneficial to incorporate heat effects and it would be fruitful to broaden the analysis to study other practically important aspects including blade wear, the impact of holes in the blades and the probability of time independence. 


\section{Conflict of Interest}

All authors declared that there is no conflict of interest involved in this work.

\section{REFERENCES}

Duffy B.R., Wilson S.K. \& Lee M.E.M. (2007). A mathematical model of fluid flow in a scraped-surface heat exchanger. Journal of Engineering Mathematics 57: 381-405. DOI: https://doi.org/10.1007/s10665-006-9116-4

Endo M., Giga Y., Gotz D. \& Liu C. (2017). Stability of a twodimensional Poiseuille-type flow for a viscoelastic fluid. Journal of Mathematical Fluid Mechanics 19(1): 17-45. DOI: https://doi.org/10.1007/s00021-016-0294-1

Fitt A.D., Lee M.E.M. \& Please C.P. (2007). Analysis of heat flow and channeling in a scraped-surface heat exchanger. Journal of Engineering Mathematics 57: 407-422. DOI: https://doi.org/10.1007/s10665-006-9097-3

Fitt A.D. \& Please C.P. (2001). Asymptotic analysis of the flow of shear-thinning food-stuffs in annular scraped heat exchangers. Journal of Engineering Mathematics 39: 345-366. DOI: https://doi.org/10.1023/A:1004834004732

Imran A., Rana M.A., Siddiqui A.M. \& Shoaib M. (2017). Flow of second grade fluid in a scraped surface heat exchanger. Journal of Food Process Engineering 40: e12393. DOI: https://doi.org/10.1111/jfpe.12393

Imran A., Siddiqui A.M. \& Rana M.A. (2016). Flow of Oldroyd 8 -constant fluid in a scraped surface heat exchanger. The European Physical Journal Plus 131: 446.

DOI: https://doi.org/10.1140/epjp/i2016-16446-2

Ishigaki Y. (2018). Global existence of solutions of the compressible viscoelastic fluid around a parallel flow. Journal of Mathematical Fluid Mechanics 204: 20732104. DOI: https://doi.org/10.1007/s00021-018-0401-6

Qin F.G.F., Chen X.D. \& Russell A.B. (2003). Heat transfer at the subcooled-scraped surface with/without phase change. AIChE Journal 49: 1947-1955.

DOI: https://doi.org/10.1002/aic.690490804
Rodriguez P.M., Derksen J.J., Van Rosmalen G.M. \& Witkamp G.J. (2009). Flow and particle motion in scraped heat exchanger crystallizers. Chemical Engineering Science 64: 5153-5161. DOI: http://10.1016/j.ces.2009.08.027

Russell A.B., Burmester S.S.H. \& Winch P.J. (1997). Characterization of shear thinning flow within a scraped surface heat exchanger. Transactions of the Institution of Chemical Engineers. Part C Food and Bioproducts Processing 75(C3): 191-197.

Smith A.A.T., Wilson S.K., Duffy B.R. \& Hall-Taylor N. (2010). Heat and fluid flow in a scraped-surface heat exchanger containing a fluid with temperature-dependent viscosity. Journal of Engineering Mathematics 68: 301-325. DOI: https://doi.org/10.1007/s10665-010-9403-y

Siddiqui A.M., Imran A., Zeb M. \& Rana M.A. (2015). Magnetohydrodynamic flow of Newtonian fluid in a scraped surface heat exchanger. Canadian Journal of Physics 93(10): 1088-1099.

DOI: https://doi.org/10.1139/cjp-2014-0195

Sun K.H., Pyle D.L., Fitt A.D., Please C.P., Baines M.J. \& HallTaylor N. (2004). Numerical study of 2D heat transfer in a scraped surface heat exchanger. Computers and Fluids 33: 869-880.

Sun K.H., Pyle D.L., Baines M.J., Hall-Taylor N. \& Fitt A.D. (2006). Velocity profiles and frictional pressure drop for shear thinning materials in lid-driven cavities with fully developed axial flow. Chemical Engineering Science 61: 4697-4706. DOI: https://doi.org/10.1016/j.ces.2006.03.005

Trommelen A.M., Beek W.J. \& Westelaken H.C. (1971). A mechanism for heat transfer in a Votator-type scrapedsurface heat exchanger. Chemical Engineering Science 26: 1987-2001.

DOI: https://doi.org/10.1016/0009-2509(71)80037-4

Wang W., Walton J.H. \& McCarthy K.L. (1999). Flow profiles of power law fluids in scraped surface heat exchanger geometry using MRI. Journal of Food Process Engineering 22: 11-27.

DOI: https://doi.org/10.1111/j.1745-4530.1999.tb00468.x 


\section{APPENDIX}

Here we have given some variable terms which are used in this work for the purpose of simplification.

$$
\begin{aligned}
& \gamma_{1}=\operatorname{Arc} \operatorname{Cosh}\left[-\frac{-p_{1 x} \beta \gamma+p_{1 x} \beta \gamma \operatorname{Cosh}\left[\frac{h p_{1 x}}{\beta}\right]+\sqrt{\beta^{2} \gamma^{2}\left(p_{1 x}{ }^{2}-2 \beta^{2} \gamma^{2}+2 \beta^{2} \gamma^{2} \operatorname{Cosh}\left[\frac{h p_{1 x}}{\beta}\right]\right) \operatorname{Sinh}\left[\frac{h p_{1 x}}{\beta}\right]^{2}}}{2 \beta^{2} \gamma^{2}\left(-1+\operatorname{Cosh}\left[\frac{h p_{1 x}}{\beta}\right]\right)}\right] \\
& \gamma_{2}=\frac{p_{1 x} \beta \gamma-p_{1 x} \beta \gamma \operatorname{Cosh}\left[\frac{h p_{1 x}}{\beta}\right]+\sqrt{\beta^{2} \gamma^{2}\left(p_{1 x}{ }^{2}-2 \beta^{2} \gamma^{2}+2 \beta^{2} \gamma^{2} \operatorname{Cosh}\left[\frac{h p_{1 x}}{\beta}\right]\right) \operatorname{Sinh}\left[\frac{h p_{1 x}}{\beta}\right]^{2}}}{2 p_{1 x} \beta \gamma-2 p_{1 x} \beta \gamma \operatorname{Cosh}\left[\frac{h p_{1 x}}{\beta}\right]} \\
& \delta_{1}=\operatorname{ArcSech}\left[-\frac{2 \sqrt{-\operatorname{Sinh}\left[\frac{(h-H) p_{2 x}}{2 \beta}\right]^{2}}}{\sqrt{-\left(\operatorname{Sinh}\left[\frac{h p_{2 x}}{\beta}\right]-\operatorname{Sinh}\left[\frac{H p_{2 x}}{\beta}\right]\right)^{2}}}\right] \\
& \delta_{2}=\frac{\beta \gamma \operatorname{Sinh}\left[\frac{(h-H) p_{2 x}}{\beta}\right]\left(\operatorname{Sinh}\left[\frac{h p_{2 x}}{\beta}\right]-\operatorname{Sinh}\left[\frac{H p_{2 x}}{\beta}\right]\right)}{p_{2 x} \sqrt{2-2 \operatorname{Cosh}\left[\frac{(h-H) p_{2 x}}{\beta}\right]} \sqrt{-\left(\operatorname{Sinh}\left[\frac{h p_{2 x}}{\beta}\right]-\operatorname{Sinh}\left[\frac{H p_{2 x}}{\beta}\right]\right)^{2}}} \\
& \phi_{1}=\operatorname{Arc} \operatorname{Cosh}\left[-\frac{-p_{3 x} \beta \gamma+p_{3 x} \beta \gamma \operatorname{Cosh}\left[\frac{H p_{3 x}}{\beta}\right]+\sqrt{\beta^{2} \gamma^{2}\left(p_{3 x}{ }^{2}-2 \beta^{2} \gamma^{2}+2 \beta^{2} \gamma^{2} \operatorname{Cosh}\left[\frac{H p_{3 x}}{\beta}\right]\right) \operatorname{Sinh}\left[\frac{H p_{3 x}}{\beta}\right]^{2}}}{2 \beta^{2} \gamma^{2}\left(-1+\operatorname{Cosh}\left[\frac{H p_{3 x}}{\beta}\right]\right)}\right] \\
& \phi_{2}=\frac{p_{3 x} \beta \gamma-p_{3 x} \beta \gamma \operatorname{Cosh}\left[\frac{H p_{3 x}}{\beta}\right]+\sqrt{\beta^{2} \gamma^{2}\left(p_{3 x}^{2}-2 \beta^{2} \gamma^{2}+2 \beta^{2} \gamma^{2} \operatorname{Cosh}\left[\frac{H p_{3 x}}{\beta}\right]\right) \operatorname{Sinh}\left[\frac{H p_{3 x}}{\beta}\right]^{2}}}{2 p_{3 x} \beta \gamma-2 p_{3 x} \beta \gamma \operatorname{Cosh}\left[\frac{H p_{3 x}}{\beta}\right]}
\end{aligned}
$$

The $Q k(k=1,2,3)$ obtained from equations (34) and (40) take the forms

$$
\begin{aligned}
Q_{1}= & -\frac{1}{12 M \Delta_{1}}\left(6\left(2 H-h_{0}-h_{1}\right) \Delta+\left(H-h_{0}\right)^{2}\left(H-h_{1}\right)^{2} l M^{3} \operatorname{Cosh}\left[\frac{H M}{2}\right]\right) \operatorname{Sech}\left[\frac{H M}{2}\right] \\
& \left(h_{0} h_{1} M\left(-12+h_{0} h_{1} M^{2}\right) \operatorname{Cosh}\left[\frac{H M}{2}\right]+12\left(h_{0}+h_{1}\right) \operatorname{Sinh}\left[\frac{H M}{2}\right]\right)\left(h_{0} h_{1} M\left(-12+h_{0} h_{1} M^{2}\right)\right. \\
& \left.\operatorname{Cosh}\left[\frac{H M}{2}\right]+12\left(h_{0}+h_{1}\right) \operatorname{Sinh}\left[\frac{H M}{2}\right]\right)+\frac{\operatorname{Tanh}\left[\frac{H M}{2}\right]}{M}
\end{aligned}
$$


$Q_{2}=\frac{1}{12 \Delta_{1}}\left(H-h_{0}\right)^{2}\left(H-h_{1}\right)^{2} l M^{2}\left(h_{0} h_{1} M\left(-12+h_{0} h_{1} M^{2}\right) \operatorname{Cosh}\left[\frac{H M}{2}\right]+12\left(h_{0}+h_{1}\right) \operatorname{Sinh}\left[\frac{H M}{2}\right]\right)$

$Q_{3}=\frac{1}{2 \Delta_{1}} h_{0} h_{1}\left(-2 H+h_{0}+h_{1}\right)\left(-12+h_{0} h_{1} M^{2}\right) \Delta+2 H\left(H^{3}\left(h_{0}+h_{1}\right)-2 H^{2}\left(h_{0}+h_{1}\right)^{2}-\right.$

$$
\left.2 h_{0} h_{1}\left(h_{0}^{2}+h_{0} h_{1}+h_{1}^{2}\right)+H\left(h_{0}+h_{1}\right)\left(h_{0}^{2}+4 h_{0} h_{1}+h_{1}^{2}\right)\right) l M^{2} \operatorname{Sinh}\left[\frac{H M}{2}\right]
$$

